\title{
An On-line Monitoring Method for Monitoring Earth Grounding Resistance Based on a Hybrid Genetic Algorithm
}

\author{
Guangxin Zhang ${ }^{1}$, *Minzhen Wang ${ }^{1}$, Xinheng Wang ${ }^{2}$, Liying Zhao ${ }^{3}$ \\ and Jinyang Zhao ${ }^{4}$ \\ ${ }^{13}$ Changchun Institute Of Technology, Changchun,jilin, 130000, China \\ ${ }^{2}$ School of Computing and Engineering, University of West London, London, UK \\ ${ }^{4}$ State Grid Fushun Power Supply Company, Fushun, Liaoning 113000, China \\ Shilin008esina.com
}

\begin{abstract}
In this paper, a method for measuring the grounding resistance of the tower without disconnecting all the down conductors is proposed for the first time in view of the shortage of manual measurement of the grounding resistance of the tower. This paper introduces the measurement model of single or multiple down conductors, and uses a hybrid genetic algorithm to comprehensively calculate the grounding resistance of all towers in the closed loop, which greatly improves the measurement accuracy. Through simulation analysis and actual measurement, it proves that the method is simple and convenient to measure, does not need to disconnect the grounding wire, and has high measurement accuracy. Compared with the clamp ammeter method, the accuracy is improved by $30 \%$. The method is applied to the on-line monitoring system of the tower grounding resistance, which can reduce the labor intensity of the line maintenance personnel, greatly improve the work efficiency and provide a basis for discovering the fault in time.
\end{abstract}

Keywords: Grounding resistance; grounding wire; measurement; hybrid genetic algorithm.

\section{INTRODUCTION}

The grounding resistance of the transmission line tower [1] is an important parameter of the tower grounding device. It is very important for the stable operation of transmission line. When there is a lightning accident, the lightning current flows through the grounding device and flows into the ground. If the grounding resistance is too high, it will produce over-voltage counterattack and cause a trip accident of the circuit [2-3]. Therefore, it is one of the important measures to ensure the operation reliability of the transmission line to measure the grounding resistance of the tower regularly and make it conform to the power industry standards [4].

At present, measurement methods mainly include fall-of-potential method[5], three-pole method[6], different-frequency method[7] and clamp ammeter method[8]. 
The fall-of-potential method requires many measurements to draw curve, this method is heavy work and difficult to operate. Three pole method is accurate, but the operation is cumbersome, ant it is necessary to arrange the voltage pole and the current pole. The grounding down conductor must be disconnected during the measurement, which greatly increases the labor intensity of the staff and has low efficiency. The different-frequency method is to inject non-power frequency signals on the basis of the three-pole method, which can effectively eliminate the power frequency interference. The injection current is small and the wiring strength is low, but it is still offline measurement, and the measurement error is greatly affected by the frequency of the input signal. The megohm meter method is simple and convenient to measure. It can be measured by clamping the grounding down conductor with a jaw. When the pole has a grounding wire, it is not necessary to disconnect the grounding down conductor. However, when there is multiple grounding down conductors, it still needs to be disconnecting other grounding down conductors and retains the measured line only, which brings inconvenience to the measurement and causes a large measurement error [9]. The common shortcoming of these methods is that manual field measurement is required. When the measurement is performed, the grounding down conductor is disconnected, which consumes a lot of manpower and material resources, and is easy to cause loose bolts or poor contact, resulting in an increase in the grounding resistance of the tower. Therefore, this paper studies a real-time monitoring method of grounding resistance that does not disconnect all grounding down conductors and has high measurement accuracy.

\section{Tower grounding resistance measurement model and measurement method without disconnecting the grounding down conductor}

When the transmission line has a lightning protection line and is directly connected to the tower to access the earth, all the towers in the same line form a parallel network through the lightning protection line, and each tower is a branch of the parallel network. The principle of measuring the unconnected grounding down conductor is to install a grounding resistance detector with the same number of grounding conductors for each tower, measure the grounding resistance of all the towers in the parallel network in time-sharing, and perform unified analysis and calculation, so as to obtain the ground resistance with higher accuracy.

The following is an example of measuring a base tower to introduce a measurement model and method for a single tower and multiple grounding down conductors.

\subsection{Static Oblique Parabolic Model}

The schematic diagram and equivalent model of the grounding resistance measurement of the single tower grounding down conductor are shown in Fig.1 and Fig.2, respectively. The measured tower and the overhead line, the adjacent tower and the grounding down conductor form a closed loop [10]. In the figure, $R_{1}$ is the grounding 
resistance of the tower to be tested, $R_{2}, R_{3} \cdots R_{n}$ are the grounding resistance of other towers in the closed loop; $R_{p 1}, R_{p 2} \cdots R_{p n}$ is the resistance of the tower itself, $L_{p 1}, L_{p 2} \cdots L_{p n}$ is the self-reactance of the tower; $R_{l 1}, R_{l 2} \cdots R_{l n}$ is used to avoid lightning resistance, $L_{l 1}, L_{l 2} \cdots L_{l n}$ is the lightning line reactance; $U_{1}$ is the output voltage of the grounding resistance detector, and $I_{1}$ is the induced loop current.

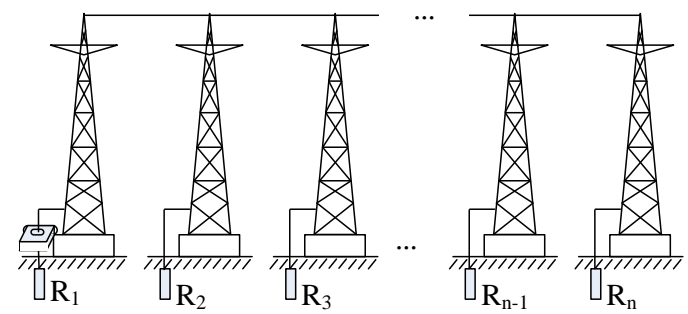

Fig. 1. Single grounding wire measurement diagram

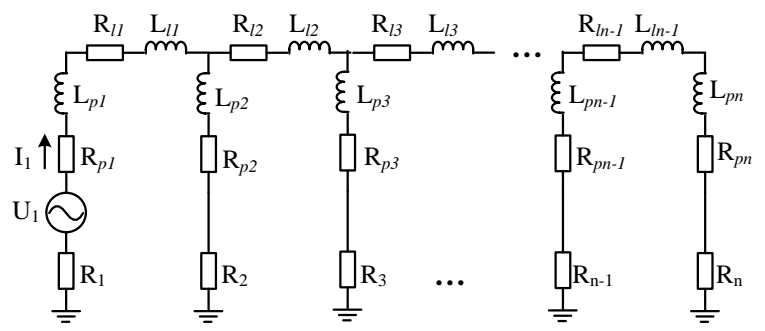

Fig.2. Single grounding wire equivalent model

Since the lightning protection line and the resistance and reactance of the tower itself are small compared with the resistance of the tower to be tested, the measurement can be neglected, so the equivalent circuit can be simplified to Fig.3, at this time, the loop resistance:

$$
R_{1}^{\prime}=R_{1}+R_{0}=\frac{U_{1}}{I_{1}}
$$

Where $R_{0}$ is the parallel value of the other tower grounding resistance, ie $R_{0}=$ $R_{2} / / R_{3} / / \cdots / / R_{n}$.

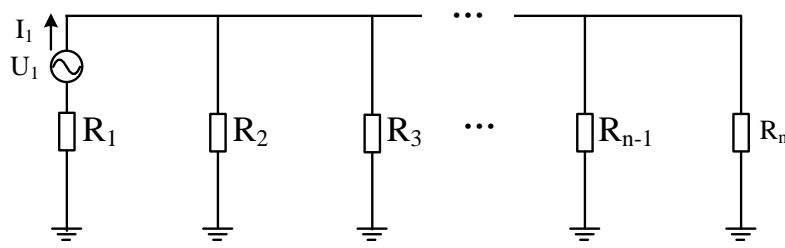

Fig.3. simplified equivalent model 
Single grounding down the line, you only need to attach the grounding resistance detector directly to the grounding line of the tower, you can measure the grounding resistance in the circuit, and then get the tower grounding resistance. The grounding resistance detector is composed of a voltage coil, a current coil and a wireless transmission module. The measuring principle is to adopt the law of electromagnetic induction, the voltage coil provides an excitation signal to the circuit under test, and induces a pulse potential $U$ on the circuit to be tested, at the potential U. Under the action, the current I will be generated in the circuit under test, $U$ and I will be measured in real time, and the resistance value of the whole measurement circuit will be calculated according to Equ. (1).

According to the specification DL/T887-2004, when the number of parallel towers meets the requirements, the parallel value of other towers adjacent to the tower to be tested is far less than the grounding resistance of the tower under test, and is ignored in the calculation., the grounding resistance of the tower to be tested is:

$$
R_{1} \approx \frac{U_{1}}{I_{1}}
$$

\subsection{Multiple grounding down conductor measurement models}

In order to realize that there is multiple grounding down conductors in the tower, it is not necessary to disconnect the grounding down conductor. In this paper, a grounding resistance detector is installed for each grounding down conductor. When measuring, each detector is connected in parallel with each other. The power supply is supplied so that the induced voltages generated by each of the grounding down conductors are exactly equal. Under the premise that the internal resistance of the grounding of the tower is the same, the induced current generated by each grounding wire is exactly the same. Fig. 4 and Fig. 5 are schematic diagrams and equivalent circuit diagrams for measuring the grounding resistance of four grounded down-conductor towers.

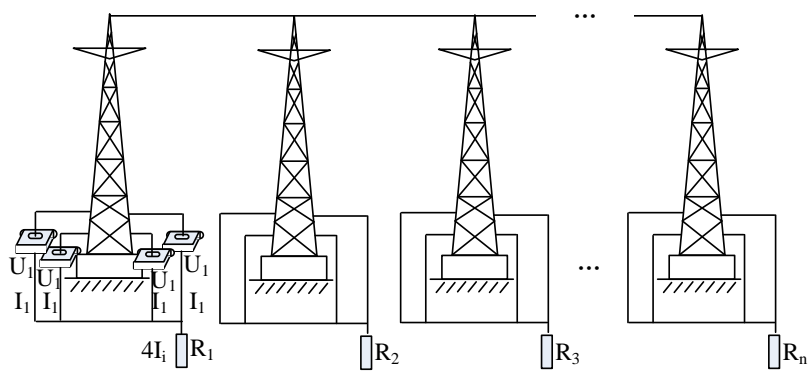

Fig. 4. Multiple grounding wire measurement schematic 


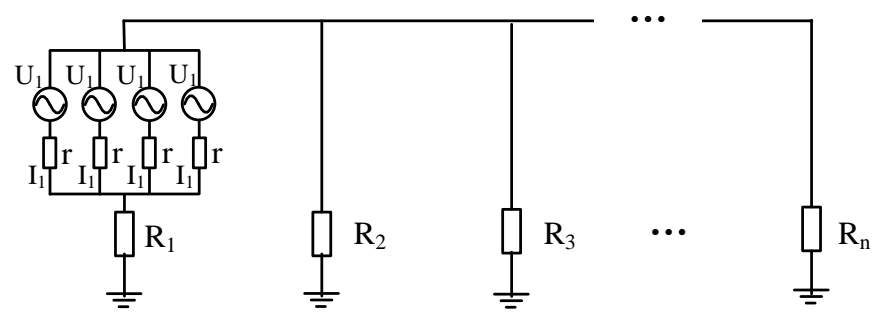

Fig. 5. Multiple grounding wire equivalent model

In Figure 4, since the induced voltage and current generated by the four grounding down conductors are exactly the same, it is only necessary to measure the induced current of one of the down conductors. Therefore, among the four detectors, only one main detector is needed, which is the same as the principle of Section 2.1. It is responsible for supplying voltage to the grounding down conductor and measuring voltage and current. The other three are auxiliary detectors, which are responsible for guiding other grounding. The line provides power. In order to ensure the same internal resistance of each grounding wire, the upper and lower ends of the detectors to which the grounding wires are connected are respectively connected by wires, so that each grounding wire is limited to a fixed area.

It can be seen from Fig. 5 that, under the premise the specification is satisfied, the method has the same voltage $U$ as the method for measuring a single down-conductor tower, and the current I flowing through the tower resistance $\mathrm{R}$ is four times the measured current. Therefore, the grounding resistance $R_{1} \approx \frac{U_{1}}{4 I_{1}}$ of the tower to be tested is $R_{1} \approx \frac{U_{1}}{\mathrm{~m} I_{1}}$ if there are $m$ grounding down conductors.

\section{Calculation method of tower grounding resistance measured without disconnecting the grounding down conductor}

Since the above measurement method ignores the lightning protection line in the loop, the tower itself and other parallel pole tower grounding resistance, there will be some error in the measurement results. In addition, in the actual measurement, there may be a local resistance increase caused by the open circuit of the ground, which also increases the measurement error of the ground resistance of the other towers. In order to reduce the error and improve the measurement accuracy, this paper considers the mutual influence between the grounding resistance of each pole in the loop, and adopts a new calculation method to analyze and calculate the grounding resistance of all the towers in the closed loop.

Measure the grounding resistance of each tower separately and bring it into Equ. (1). 


$$
\left\{\begin{array}{c}
\mathrm{R}_{1}^{\prime}=R_{1}+\frac{1}{\frac{1}{R_{2}}+\frac{1}{R_{3}}+\cdots+\frac{1}{R_{i}}+\cdots+\frac{1}{R_{n}}} \\
\mathrm{R}_{2}^{\prime}=R_{2}+\frac{1}{\frac{1}{R_{1}}+\frac{1}{R_{3}}+\cdots+\frac{1}{R_{i}}+\cdots+\frac{1}{R_{n}}} \\
\cdots \cdots \\
\mathrm{R}_{i}^{\prime}=R_{i}+\frac{1}{\frac{1}{R_{1}}+\cdots \frac{1}{R_{i-1}}+\frac{1}{R_{i+1}}+\cdots+\frac{1}{R_{n}}} \\
\cdots \cdots \\
\mathrm{R}_{n}^{\prime}=R_{n}+\frac{1}{\frac{1}{R_{1}}+\frac{1}{R_{2}}+\cdots+\frac{1}{R_{i}}+\cdots+\frac{1}{R_{n-1}}}
\end{array}\right.
$$

Where: $\mathrm{R}_{i}^{\prime}$ is the measured value of the grounding resistance of the $i$ th base tower in the parallel network. When the tower has one grounding down conductor, $\mathrm{R}_{i}^{\prime}=\frac{U_{i}}{I_{i}}$; when the tower has $m$ grounding down conductors, $\mathrm{R}_{i}^{\prime}=\frac{U_{i}}{\mathrm{~m} I_{i}} ; U_{i} 、 I_{i}$ are the voltage and current detected by the ground resistance detector; $R_{i}$ is the value of the tower grounding resistance to be calculated.

By solving the Equ. (3), the tower grounding resistances $R_{1}, R_{2} \cdots R_{n}$ can be obtained. Equ. (3) is a system of nonlinear equations with $n$ variables and $n$ equations ( $n$ $\geq 4$ ). It is very difficult to solve directly. It needs to be transformed into extreme value problems. Classical algorithms (such as Newton method, gradient method and steepest descent Method, etc.) or genetic algorithm for calculation are used. The classical algorithm has strong local search ability and fast convergence speed, and can obtain the local optimal solution. However, its convergence is related to the selection of the initial point. The initial point quality directly affects the accuracy of the algorithm, but the initial selection is good. The point is very difficult. Genetic algorithm [11] (GA) is a randomized search method for simulating the evolutionary process of biological survival of the fittest. It has a strong global search ability and can quickly obtain the optimal solution range, but its local convergence ability is weak. Equ. (3), genetic algorithm is not dominant compared with the classical algorithm, usually only find the suboptimal solution of the problem.

Combining the advantages and disadvantages of classical algorithm and genetic algorithm, this paper proposes a hybrid genetic algorithm [12] which combines classical algorithm (conjugate gradient method [13]) and genetic algorithm to solve nonlinear Equ. (3). The algorithm fully inherits the global search ability of the genetic algorithm and the local search and fast convergence ability of the classical algorithm, which can effectively improve the convergence speed and the accuracy of the solution, and quickly and accurately find the global optimal solution.

For the convenience of calculation, the Equ. (3) can be simply expressed as:

$$
\left\{\begin{array}{c}
f_{1}\left(R_{1}, R_{2}, R_{3}, \cdots, R_{n}\right)=\mathrm{R}_{1}^{\prime} \\
f_{2}\left(R_{1}, R_{2}, R_{3}, \cdots, R_{n}\right)=\mathrm{R}_{2}^{\prime} \\
\cdots \cdots \\
f_{i}\left(R_{1}, R_{2}, R_{3}, \cdots, R_{n}\right)=\mathrm{R}_{i}^{\prime} \\
\cdots \cdots \\
f_{n}\left(R_{1}, R_{2}, R_{3}, \cdots, R_{n}\right)=\mathrm{R}_{n}^{\prime}
\end{array}\right.
$$


Where, $f_{i}\left(R_{1}, R_{2}, R_{3}, \cdots, R_{n}\right)=R_{i}+\frac{1}{\frac{1}{R_{1}}+\cdots \frac{1}{R_{i-1}}+\frac{1}{R_{i+1}}+\cdots+\frac{1}{R_{n}}}$

The idea of using a hybrid genetic algorithm to solve the Equ.(4) is: First, construct the objective function of the genetic algorithm, and transform the Equ.(4) into the extremum problem, that is, find an $R$, so that the value of the Equ. (4) is the smallest. When the minimum value approaches to 0 , the corresponding $R$ is the solution of the system of equations, and the objective function is shown in Equ. (5). Then, the selection, crossover and mutation operations of the genetic algorithm are performed to find a point near the most advantageous point (population center point). Finally, the population center point obtained by the genetic algorithm is used as the initial point of the conjugate gradient method for secondary optimization. Find the minimum value of the objective function and find the optimal solution. The specific steps are shown in Fig. 6.

$$
\left\{\begin{array}{c}
\text { find: } R=\left(R_{1}, R_{2}, R_{3}, \cdots, R_{n}\right) \\
\text { s.t.: } \operatorname{minf}(R)=\min \sqrt{\sum_{i=1}^{n}\left(f_{i}(R)-\mathrm{R}_{i}^{\prime}\right)^{2}}
\end{array}\right.
$$

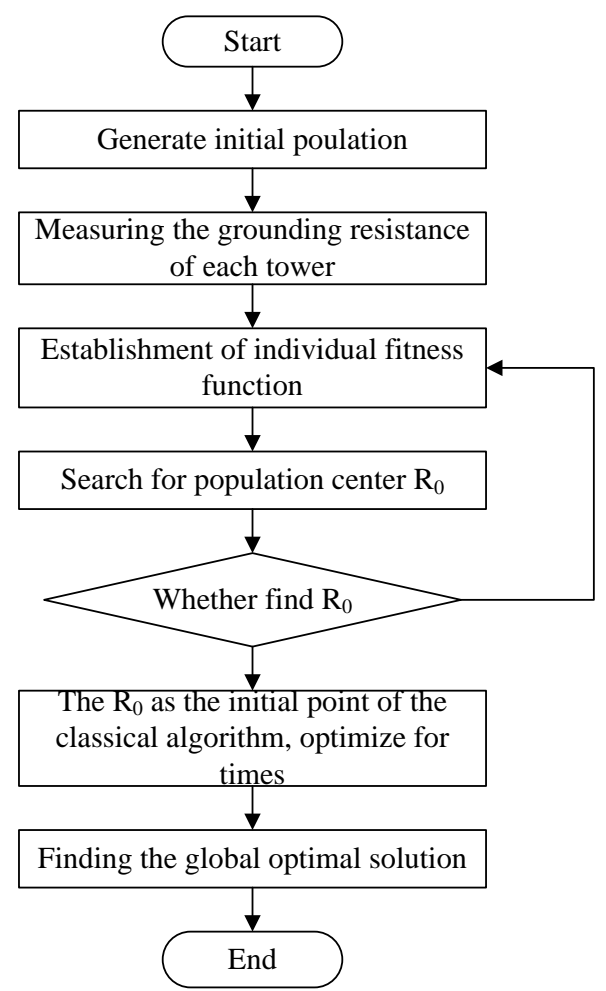

Fig. 6. Hybrid genetic algorithm flow of grounding resistance 


\section{MODEL SIMULATION AND APPLICATION}

In order to verify the accuracy of the method for measuring the grounding resistance of the tower and the accuracy of using the hybrid genetic algorithm to calculate the grounding resistance, a grounding resistance measurement simulation model was built. As shown in Fig. 3 and Fig. 5, five towers were built. In the simulation, the excitation voltage is set to $1 \mathrm{~V}$ and the frequency is $1582 \mathrm{~Hz}$.

\subsection{Simulation analysis of grounding resistance measurement method without disconnecting grounding wire}

For the case that the tower has a single root and multiple grounding down conductors, the traditional method - the clamp ammeter method and the unconnected ground down conductor measurement method are used to measure the grounding model parameters $\mathrm{U}_{i}$ and $\mathrm{I}_{i}$, and calculate according to Equ. (2). The grounding resistance is approximated by $\mathrm{R}_{i}^{\prime}$, and the measurement results are shown in Table 1 .

Table 1. Comparison of traditional methods and measured values of this research method

\begin{tabular}{|c|c|c|c|c|}
\hline \multirow{2}{*}{$\begin{array}{l}\text { Grounding } \\
\text { pole number }\end{array}$} & \multicolumn{2}{|c|}{ Single ground wire } & \multicolumn{2}{|c|}{ Multiple grounding wires } \\
\hline & $\begin{array}{l}\text { clamp amme- } \\
\text { ter method }\end{array}$ & $\begin{array}{l}\text { Do not disconnect } \\
\text { the ground wire }\end{array}$ & $\begin{array}{l}\text { the clamp am- } \\
\text { meter method }\end{array}$ & $\begin{array}{l}\text { Do not disconnect } \\
\text { the ground wire }\end{array}$ \\
\hline 1 & 6.35 & 6.35 & 4.18 & 4.18 \\
\hline 2 & 8.71 & 8.70 & 7.25 & 7.24 \\
\hline 3 & 12.3 & 12.31 & 5.32 & 5.32 \\
\hline 4 & 5.60 & 5.60 & 10.51 & 10.5 \\
\hline 5 & 6.35 & 6.34 & 7.26 & 7.25 \\
\hline
\end{tabular}

According to the comparison results in Table 1, the grounding resistance values of the towers measured by the two methods are basically the same, that is, in the same environment, the resistance value measured by the grounding resistance measurement method of the tower without disconnecting the grounding down conductor can be compared with the clamp ammeter method. Comparable, and there is no need to disconnect the ground lead.

\subsection{Accuracy analysis of the calculation method of the grounding resistance of the tower without measuring the grounding wire}

Simulation analysis at Section 4.1 is only for the purpose of verifying the accuracy of the measurement method and directly using the comparative analysis of the measurement results. In this section, the direct measurement results are compared with the results calculated by the hybrid genetic algorithm, and the accuracy of the calculation results is verified. The error comparison is shown in Table 2. 
Table 2. Comparison of error calculation results using/without genetic algorithm

\begin{tabular}{|c|c|c|c|c|c|}
\hline \multirow{2}{*}{$\begin{array}{c}\text { Grounding } \\
\text { pole number }\end{array}$} & Theoretical & \multicolumn{2}{|c|}{ Unused genetic algorithm } & \multicolumn{2}{c|}{ Used genetic algorithm } \\
\cline { 3 - 6 } & value $(\Omega)$ & Calculated $(\Omega)$ & error $(\%)$ & Calculated $(\Omega)$ & error $(\%)$ \\
\hline 1 & 9 & 11.55 & 28.33 & 9.14 & 1.56 \\
\hline 2 & 8 & 9.21 & 15.13 & 8.03 & 0.37 \\
\hline 3 & 10 & 11.37 & 13.70 & 10.01 & 0.10 \\
\hline 4 & 7 & 8.62 & 23.14 & 7.02 & 0.29 \\
\hline 5 & 13 & 15.87 & 22.08 & 13.21 & 1.62 \\
\hline
\end{tabular}

In order to more intuitively represent the error situation, the error curve of the mixed and used genetic algorithm is drawn using Matlab as shown in Fig.7.

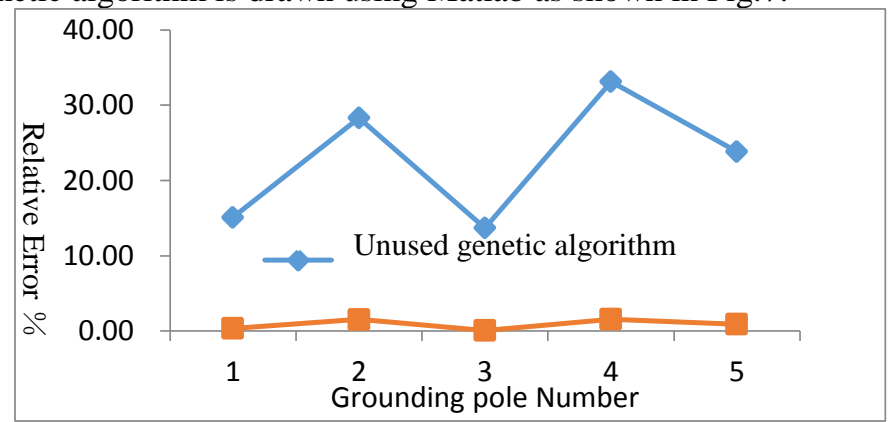

Fig. 7. Relative error curve of ground resistance

It can be seen from Fig. 6 that the grounding resistance error is relatively large without using the genetic algorithm, and the error of the resistance value calculated by the hybrid genetic algorithm is greatly reduced. In the unconnected grounding down-line measurement model, the hybrid genetic algorithm is used to calculate the grounding. The resistance can greatly improve the measurement accuracy of the tower grounding resistance.

The method for measuring the grounding resistance of the poles of the uninterrupted grounding wire studied in this paper has been applied to the real-time monitoring system of the ground resistance of the transmission line, and it has been put into use in the state grid Yanbian power supply company $66 \mathrm{kV}$ Yanxijiayi line 2\# 11\#, and the state grid Dandong power supply company $66 \mathrm{kV}$ Langqian line $1 \# \sim 6 \#$. Since the installation, the operation has been stable, and several abnormalities in the grounding resistance of the tower have been found. For example, Langqian line $3 \#$ tower grounding pole grounding resistance being very high, nearly $300 \Omega$, and other grounding resistance values are normal, which indicates that there is a bad contact condition in the grounding pole of the $3 \#$ pole tower. The staff found the hidden danger of the line in time according to the measurement result and avoided the occurrence of lightning strikes. The long-term operation results show that the method is convenient to measure, does not need to disconnect the grounding wire, and has high precision, which can completely replace the traditional pole tower grounding resistance measurement method. It provides great convenience for line maintenance personnel and provides technical support for timely detection of hidden dangers. 


\section{CONCLUSION}

In this paper, the measurement method of the grounding resistance of the transmission line tower is studied, and the grounding resistance of the tower is measured without any disconnection. The method has obvious advantages for the measurement of the tower with multiple grounding down conductors. In order to improve the measurement accuracy, the mutual influence between adjacent towers in the same parallel network is considered, the grounding resistance of each tower is measured, the grounding resistance equations are constructed, and the grounding resistance is calculated by the hybrid genetic algorithm, which greatly improves the measurement accuracy. Through simulation analysis and practical application, the method studied in this paper is simple in operation and high in measurement accuracy. It can be used in online monitoring system, replacing traditional manual measurement methods, greatly improving the labor efficiency of workers, and the application prospect is very broad.

\section{References}

1. Lin Jiehuan, F.: Three - pole method and clamp - meter method for measuring earthing resistance of transmission line. Electrical engineering and automation (30),1-2(2016).

2. Liao Fuwang, F., Huang Weixian, S.: Study on new model and test method of ground parameter of line pole tower. Power and Electrical Engineering 29(4), 1-4(2009).

3. Xu Wei, F., Liu Xun,S.: Based on ATP-EMTP, the calculation model of impact grounding resistance of tower ground body. electric power construction 31(5),22-25(2010).

4. Wu Hao, F.: Accurate measurement method and experimental study of earthing resistance of poles and towers. Chongqing university,1-2(2016).

5. Wu yanwu, F., Xie baoyong, S.: Effect of the length of the test line on the measurement of grounding resistance by potential drop method. New technology and new products in China 1(2),32-33(2016).

6. Yang meng, F.: Research on measurement method of ground resistance of transmission line poles and towers. new technology and new product in China (9),78(2015).

7. Jia chao, F., Hu zhijian,S.: Measurement of grounding resistance of grounding grid by multiple synchronous frequency.Power automation equipment (4), 167-172(2015).

8. Zhou Qingdong, F.: Comparison and analysis of ground resistance measurement of two commonly used transmission line poles and towers. Electrical measurement 12(9),4849(2012).

9. FlAVIO GALlianA, F., PIER PAOLO CAPRA, S.: Traceable technique to calibrate clamp meters in AC current from 100 to 1500 A. IEEE Trans on Power System 61(9), 2512-2513(2012).

10. Guo Kun, F.: Study on measuring method of ground resistance of transmission line tower. North China electric power university, 11-15(2008).

11. Tian Qiaoyu, F., Gu zhongbi, S.: Solving nonlinear equations based on hybrid genetic algorithm. Computer technology and development 17 (3),10-12(2007).

12. Xing Hongyan, F., He Guixian S.: Application of hybrid genetic algorithm in ground resistance measurement. Journal of electronic measurement and instrument 30(9),13891396(2016).

13. Li Jiongcheng, F.,Wang yangyang, S.: Fast convergence of hybrid genetic algorithm. Computer engineering and design 35(2),686-689 (2014). 